\title{
Sub-diffraction surface topography measurement using a microsphere- assisted Linnik interferometer
}

\author{
Paul C. Montgomery, Sylvain Lecler, Audrey Leong-Hoï, Stéphane Perrin and Pierre Pfeiffer \\ Laboratoire des Sciences de l'Ingénieur, de l'Informatique et de l'Imagerie (ICube), Unistra-CNRS, \\ UMR 7357, 23 rue du Loess, 67037 Strasbourg cedex, France.
}

\begin{abstract}
Microscopic surface topography measurement is an important aspect of industrial inspection. Optical and near field scanning techniques are increasingly replacing the use of the traditional mechanical stylus since they provide better lateral resolutions and higher measurement speeds. The main far field optical techniques used are interference microscopy and confocal microscopy, with the advantages of having larger fields of view and higher measurement speeds. Interference microscopy is now widely used, mainly because of its nanometric axial measurement sensitivity and its ease of use but suffers from a limitation in lateral resolution of about $\lambda / 2$ due to diffraction. A new technique for high resolution $2 \mathrm{D}$ imaging using a microsphere placed on the sample has been recently combined with interferometry by several groups to greatly improve the lateral resolution. In this paper we present some of our own first results using glass microspheres with a white light Linnik interferometer and demonstrate a lateral resolution of $\lambda / 4$ and an axial measurement sensitivity of several $\mathrm{nm}$. Results are shown on calibrated square profile gratings with periods down to $400 \mathrm{~nm}$, with a minimum feature size of $200 \mathrm{~nm}$ and a height of $148 \mathrm{~nm}$ and a field of view of several $\mu \mathrm{m}$. While these features are not visible directly with the microscope objective, they become observable and measurable through the microsphere. An analysis using rigorous electromagnetic simulations is also given to help better understand the imaging properties of the technique. These first experimental and simulation results clearly indicate that this is an important new technique that opens new possibilities for surface metrology with a lateral resolution well beyond the diffraction limit.
\end{abstract}

Keywords: coherence scanning interferometry; Linnik interferometer; super-resolution; microsphere; photonic jet; surface metrology

\section{INTRODUCTION}

Microscopic surface topography measurement is an important aspect of industrial inspection. Optical and near field scanning techniques are increasingly replacing the use of the traditional mechanical stylus. The reasons for this are that they provide better lateral resolutions and/or higher measurement speeds. While near field techniques provide lateral resolutions down to several $\mathrm{nm}$, they are fundamentally limited in measurement speed by the need for lateral tip scanning. The different far field optical techniques typically used are interference microscopy and confocal microscopy. The advantages of remaining in the far field are the larger fields of view of hundreds of $\mu \mathrm{m}$ to several mm and the higher measurement speeds, ranging from several seconds to several minutes depending on the volume measured ${ }^{1}$. The main disadvantage compared with near field techniques is the lateral resolution that is limited by diffraction to about $\lambda / 2$, or between $200 \mathrm{~nm}$ to $500 \mathrm{~nm}$ in the visible region. The lateral resolution can be calculated from the criterion $R c=K \lambda / N A$, where $R c$ is the distance between two point sources, $N A$ is the numerical aperture, with $N A=n . \sin \theta$ and $\theta$ is the half angle of the light cone entering the objective, $n$ is the refractive index of the object-space and $K$ is 0.61 or 0.47 depending on whether the resolution definition comes from the Rayleigh criterion or Sparrow criterion respectively ${ }^{2}$. Improvements in lateral resolution to better than $100 \mathrm{~nm}$ have been achieved by means of increasing the synthetic aperture, in the case of structured illumination microscopy (SIM) by using a projected high resolution $\operatorname{grid}^{3}$, and in tomographic diffractive microscopy (TDM) by changing the solid angle of illumination ${ }^{4}$. 
One of the most commonly used far field techniques is interference microscopy, mainly because of its very high axial measurement sensitivity of less than $1 \mathrm{~nm}$ and its ease of use. Nonetheless, one disadvantage remains the limited lateral resolution which is over two orders of magnitude worse than the axial measurement sensitivity. In the field of high resolution 2D imaging, an important technique introduced several years ago is the use of a microsphere placed on the sample in front of the microscope objective, with a claimed lateral resolution of $50 \mathrm{~nm}$ in the best conditions ${ }^{5}$. More recently, in 2016, the microsphere technique was successfully combined with interferometry by several groups in a major step forward in reducing the gap between lateral resolution and axial measurement sensitivity ${ }^{6-8}$. In this paper we present some of our own first results of combining the microsphere technique with a white light Linnik interferometer and demonstrate a lateral resolution of $\lambda / 4$ and an axial measurement sensitivity of several $\mathrm{nm}$.

Results are shown on the measurement of calibrated square profile gratings in air with periods ranging from $400 \mathrm{~nm}$ to 1.2 $\mu \mathrm{m}$, heights from $50 \mathrm{~nm}$ to $150 \mathrm{~nm}$ and a field size of up to $4 \mu \mathrm{m}$ through $24 \mu \mathrm{m}$ diameter microspheres. While the smaller period structures are not visible directly by the objective, they become visible and measurable through the microsphere. An analysis using rigorous electromagnetic simulations is also given to help understand the imaging process of the technique. These first experimental and simulation results clearly indicate that this is a significant new technique that opens new possibilities for surface metrology well beyond the diffraction limit.

\section{3D MICROSCOPE SYSTEM}

\subsection{Linnik measurement system}

The system for combining interferometry with microsphere imaging has been developed around a modified Leitz-Linnik interference microscope ${ }^{9}$ with identical 50X $(\mathrm{NA}=0.85)$ objectives (figure 1$)$. Two light sources are available, the first, an incandescent lamp with a central wavelength of $\lambda_{0}=760 \mathrm{~nm}\left(\mathrm{LS}_{1}\right)$ and the second, a white light LED with a central wavelength of $\lambda_{0}=560 \mathrm{~nm}\left(\mathrm{LS}_{2}\right)$. The sample is placed on a PIFOC piezoelectric nanopositioner (with closed loop capacitive position sensor) to provide the fringe scanning along the optical axis over the depth of the sample surface to be measured. The imaging camera used is a monochrome CMOS camera (PhotonFocus MV1-D2048-96-G2) with a Giga Ethernet connection. A PC equipped with an Intel ${ }^{\circledR}$ Xeon ${ }^{\circledR}$ CPU processor $(2.40 \mathrm{GHz}, 8$ Go RAM $)$ under a Windows 7 (64 bits) operating system is used to control the system.

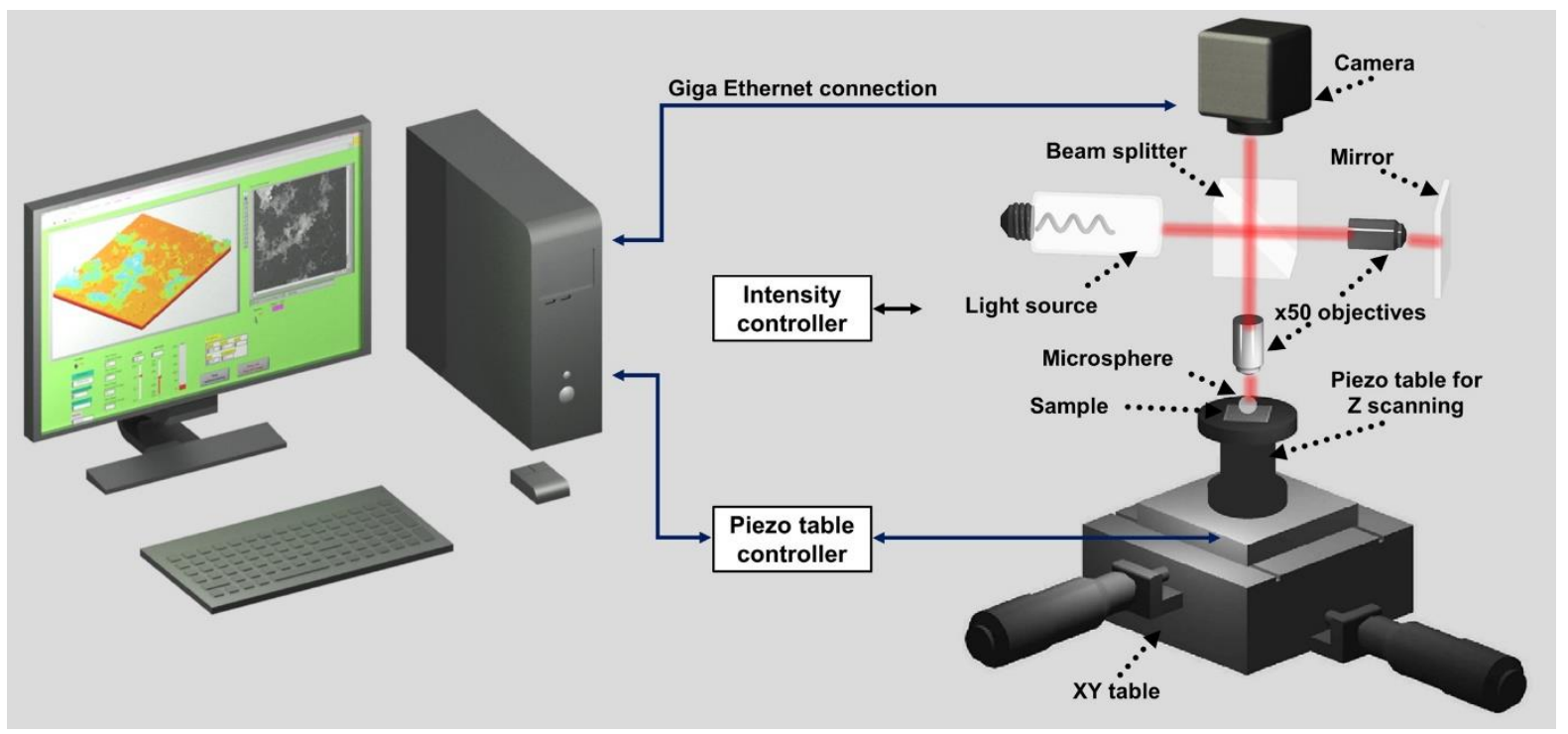

Figure 1. Layout of the Leitz-Linnik microscope-based system for combining interferometry with a microsphere.

The glass microspheres have a diameter of $24 \mu \mathrm{m}$, an optical index of $\mathrm{n}=1.5$ and a size dispersion of $5 \%$ (from Cospheric). These are placed directly on the sample between the objective and the region of interest to be measured. The image of the 
sample is first formed by direct observation of the sample surface through the microsphere. The interference fringes are then found by adjusting the difference in optical path of the two arms of the Linnik interferometer. In other terms, the focal gate and coherence gates are aligned ${ }^{10}$.

\subsection{Control and image processing software}

In-house developed software is used for the control and analysis of the system, using LabVIEW (version 2014, 64 bits, from National Instruments) combined with the IMAQ Vision module. A modified version of the 5 phase step algorithm $(\lambda / 2)$ is used with image averaging at each step (up to 10 images) to make the phase measurement. The central region of interest of the measurement is isolated and a standard phase unwrapping routine employed to remove the phase discontinuities. The curved form due to the presence of the microsphere is removed and light ( $3 \times 3$ pixels) median/low pass filtering is used to reduce the camera noise.

\subsection{AFM measurements}

The surface topography was also measured using a Park XE70 AFM microscope placed in a vibration isolation chamber and working in the non-contact mode. The tip used was a noncontact high frequency point probe with a tip radius of $2 \mathrm{~nm}$ and a width of $10 \mathrm{~nm}$ at a distance of $100 \mathrm{~nm}$ from the tip. The lateral resolution is $\mathrm{R}_{\text {lat }}=0.012 \mu \mathrm{m}$ to $0.195 \mu \mathrm{m}$ depending on the field size, which is up to $50 \times 50 \mu \mathrm{m}$ for $256 \times 256$ pixels.

\section{3D RESOLUTION STANDARD AND FRINGE OBSERVATION}

\subsection{Choice of grating standard}

For the experimental demonstration of 2D super-resolution on structures below $0.5 \mu \mathrm{m}$ in size, different samples have been used in the literature such as CD-ROM surface patterns ${ }^{11}$, the periodic lines of a Blu-Ray DVD disk (spacing of $112 \mathrm{~nm}$ ), the pores in an anodic aluminum oxide (AAO) sample (average diameter of $50 \mathrm{~nm}$ ) and individual adenoviruses (approximately $75 \mathrm{~nm})^{5,12}$. To demonstrate 3D super-resolution, the choice of the sample is not simple. 3D structures that have been proposed in the literature consist of the periodic line structures of a stripped Blu-Ray DVD disk (spacing of 112 $\mathrm{nm})^{6,7}$, microelectronic structures in a CPU chip ${ }^{5}$, a VLSI STR10-1000P silicon test pattern, composed of $5 \mu \mathrm{m}$ wide, 100 $\mathrm{nm}$ high square structures with a pitch spacing of $5 \mu \mathrm{m}^{4}$ and periodic square grating 3D test patterns ${ }^{8}$. In the present work, the latter type of reference gratings was used (prototype RS-N gratings from SiMETRICS GmbH) consisting of a series of sub-gratings with pitch values varying from $300 \mathrm{~nm}$ to $6 \mu \mathrm{m}$. The structures are dry etched up to a nominal depth of 190 $\mathrm{nm}$ in a $10 \mathrm{~mm} \times 10 \mathrm{~mm} \mathrm{Si}$ wafer. In this work, results are given for the gratings with pitch values of $1.2 \mu \mathrm{m}, 0.6 \mu \mathrm{m}$ and $0.4 \mu \mathrm{m}$. The incandescent lamp $\left(\mathrm{LS}_{1}\right)$ was used for the first two gratings and the LED $\left(\mathrm{LS}_{2}\right)$ for the smallest grating.

\subsection{Observation of gratings with Zeiss reflection microscope}

The super-resolution effect is first demonstrated by placing the RS-N grating sample under a Zeiss reflection microscope. The results in figure 2 show the comparison of the images focused on the grating directly with the objective $(\mathrm{x} 10$, NA = 0.2 ) in (a) and then through a series of $24 \mu \mathrm{m}$ diameter microspheres in (b). It can be observed that only the $6 \mu \mathrm{m}, 4 \mu \mathrm{m}$, $3 \mu \mathrm{m}$ and $2 \mu \mathrm{m}$ pitch gratings can be resolved directly without the microsphere, the $1.2 \mu \mathrm{m}$ pitch being below the resolution limit of the objective. Focusing through the microspheres, both the $1.2 \mu \mathrm{m}$ and the $0.6 \mu \mathrm{m}$ pitch gratings can be resolved in the virtual image plane through the microspheres.

The images in figure 3 are the results of imaging through a $24 \mu \mathrm{m}$ diameter microsphere placed on the $0.6 \mu \mathrm{m}$ pitch grating using the Leitz-Linnik microscope in air with white light illumination $\left(\mathrm{LS}_{1}\right)$. Figure 3(a) shows a 2D super-resolved image of the grating through the microsphere and figure 3(b) the same but with the white light interference fringes added. These fringes can be observed to be circular and to be modified by the surface topography of the $3 \mathrm{D}$ grating, demonstrating that the phase of the reflected light is perturbed by the magnified grating surface. 


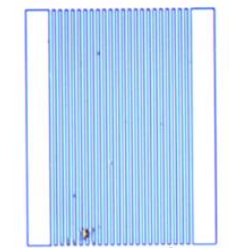

$4 \mu \mathrm{m}$

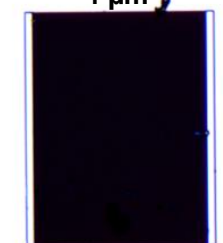

$0.8 \mu \mathrm{m}$

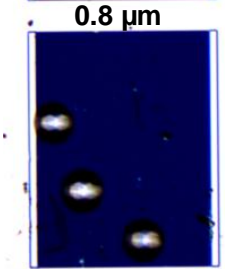

$1.2 \mu \mathrm{m}$

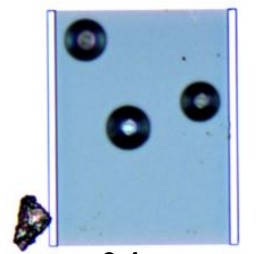

$0.4 \mu \mathrm{m}$

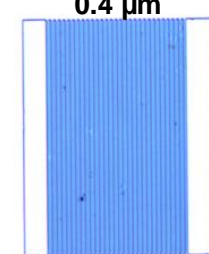

$3 \mu \mathrm{m}$

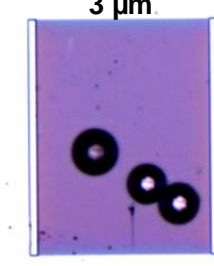

0.3. $\mu \mathrm{m}$

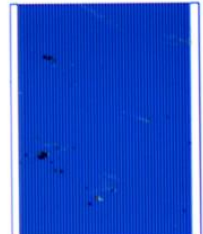

$2 \mu \mathrm{m}$

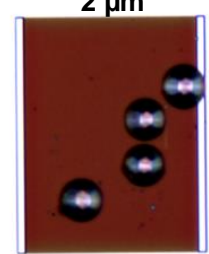

$0.6 \mu \mathrm{m}$

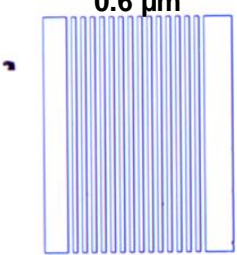

$6 \mu \mathrm{m}$
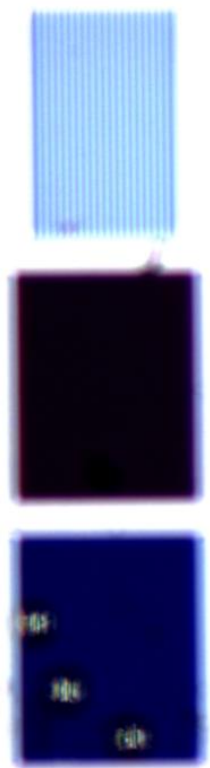

(b) Focused through microspheres.

(a) Focused on grating.
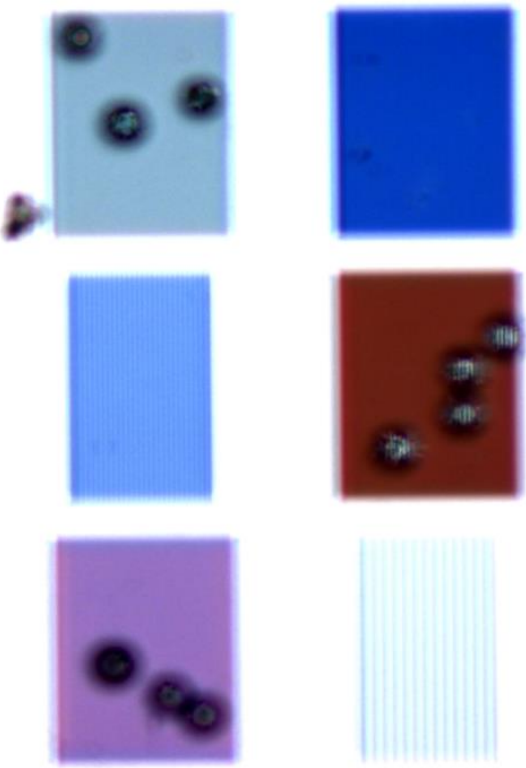

Figure 2. Reference gratings RS-N observed with a Zeiss optical microscope in white light with an objective of x10 $(\mathrm{NA}=0.2)$ directly and through $24 \mu \mathrm{m}$ diameter microspheres.

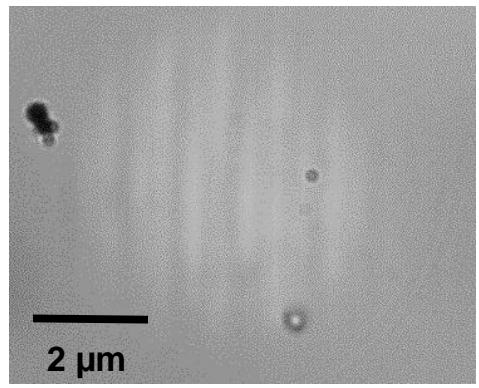

(a) Super-resolved image of the grating through the microsphere.

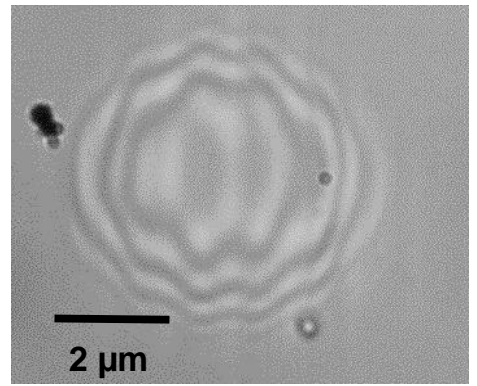

(b) Fringes superimposed on the image of the grating through the microsphere.

Figure 3. Imaging of a $0.6 \mu \mathrm{m}$ pitch grating through the $24 \mu \mathrm{m}$ diameter microsphere in the Leitz-Linnik microscope using $\operatorname{LS}_{1}\left(\lambda_{0}=760 \mathrm{~nm}\right)$.

\section{3D MEASUREMENTS OF SI GRATINGS}

In this section, results are given of the comparison of the 3D measurements of the gratings made with the Leitz-Linnik microscope and with AFM. The results of depth measurements, magnification and resolution are summarized in Table 1 at the end of the section. The magnification obtained through the microspheres is determined by the ratio between the period measured through the microspheres and the theoretical period value. The lateral resolution is calculated using the Sparrow criterion for the direct view using the objective and from half the grating period value for the measurements 
through the microsphere. Although the NA for the Linnik objective is quoted to be 0.85 , the NA for the system used is nearer to 0.3 , as calculated using the grating standard.

\subsection{Measurements of the $1.2 \mu \mathrm{m}$ pitch grating}

Results on the $1.2 \mu \mathrm{m}$ pitch grating are given in figure 4 . The 3D shape can just about be measured using the classical technique with the Leitz-Linnik with the objective alone using light from the incandescent lamp (figure 4(d),(e),(f)) but gives a measured depth of only $30 \mathrm{~nm}$, compared with the depth of $151 \mathrm{~nm}$ measured by AFM. The main reason for this is probably due to the measurement being made near to the lateral resolution limit of the objective/microsphere system, in which it is well known that the depth of structures near to this limit is reduced ${ }^{2}$.

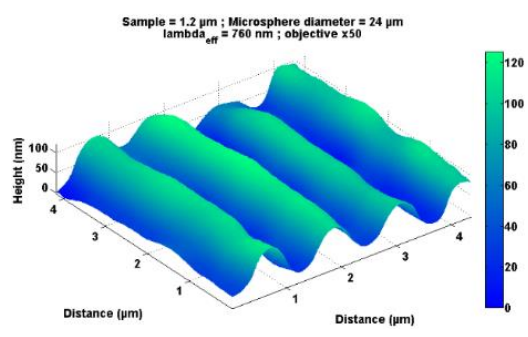

(a) $3 \mathrm{D}$ view through the microsphere.

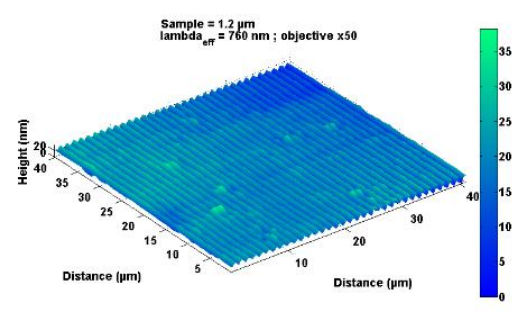

(d) 3D direct measurement.

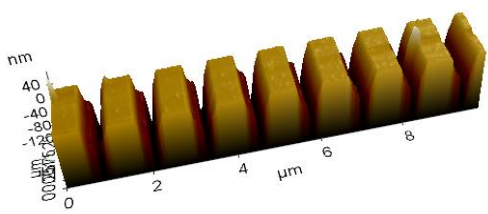

(g) 3D view using AFM.

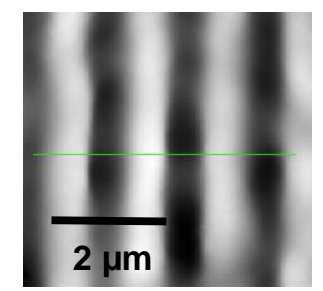

(b) Topography.

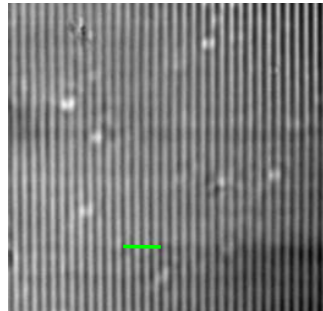

(e) Topography.

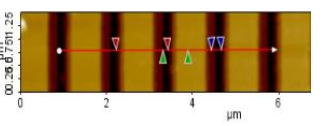

(h) Topography.

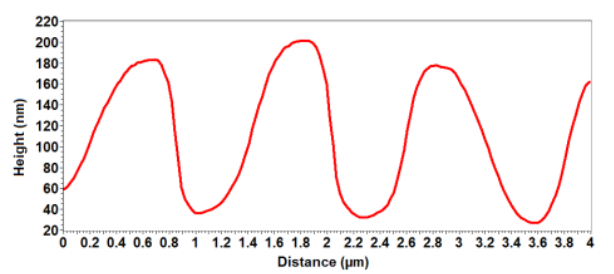

(c) Profile from (b): average height $=150$ nm.

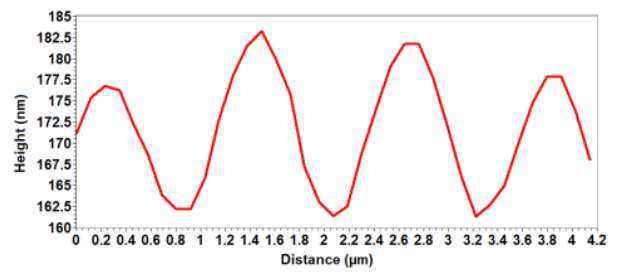

(f) Profile from (e): average height $=30$ $\mathrm{nm}$.

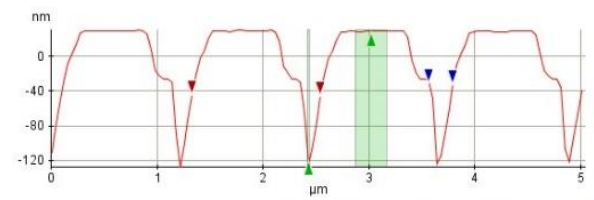

(i) Profile from (h): height between green arrows $=153 \mathrm{~nm}$.

Figure 4. Comparison of measurements of the $1.2 \mu \mathrm{m}$ pitch grating through the $24 \mu \mathrm{m}$ diameter microsphere in white light $\left(\mathrm{LS}_{1}, \lambda_{0}=760 \mathrm{~nm}\right)$ with the 50X Leitz-Linnik microscope and using AFM.

The 3D measurement through the $24 \mu \mathrm{m}$ diameter microsphere (figure 4(a),(b),(c)) show that the grating is magnified by a factor of 4.5 and that the 3D profile is much squarer (figure 4(c)). The measured depth is now $150 \mathrm{~nm}$, much closer to the value obtained by AFM (figure $4(\mathrm{~g})$, (h), (i)) revealing a pitch of $1.21 \mu \mathrm{m}$ and a total depth of around $151 \mathrm{~nm}$. Nonetheless the depth profile measured can be observed to be not square but with sidewalls that appear inclined and with a V-shaped structure (figure 4(i)). The differences in the measured profiles with those through the microsphere could be either due to a lack of lateral resolution in the latter case or to slight variations in the profiles between the different positions measured with the two techniques. 


\subsection{Measurements of the $0.6 \mu \mathrm{m}$ pitch grating}

Since the $0.6 \mu \mathrm{m}$ pitch grating cannot be resolved directly, the 3D measurements were not possible just with the objective in the Leitz-Linnik. On the contrary, this grating could be measured through the microsphere, giving a magnification of x3.65 and a measured depth of $46 \mathrm{~nm}$ (figure 5(a), (b), (c)). The AFM measurements reveal a pitch of $0.633 \mu \mathrm{m}$ and a total depth of around $58 \mathrm{~nm}$ (figure $5(\mathrm{~d}),(\mathrm{e}),(\mathrm{f}))$.

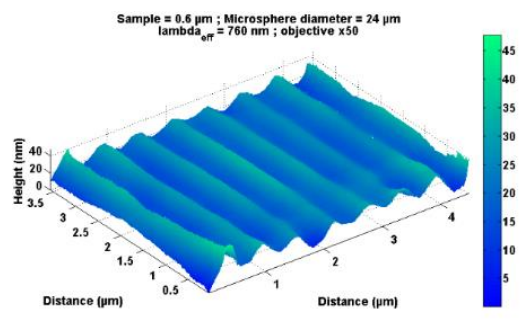

(a) 3D measurement through the microsphere.

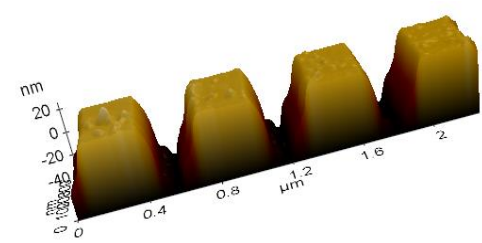

(d) 3D view using AFM

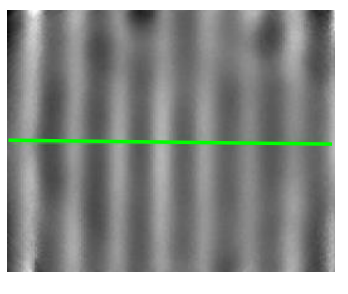

(b) Topography.

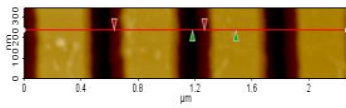

(e) Topography

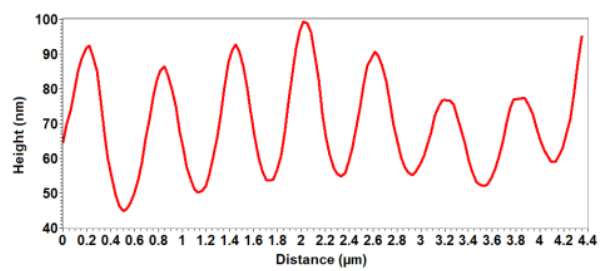

(c) Profile from (b): average height $=46$ nm.

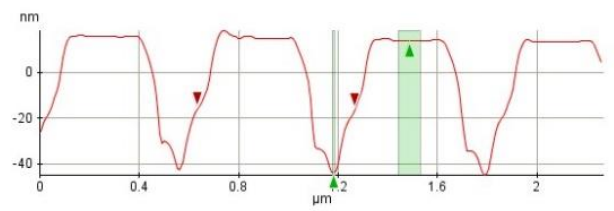

(f) Profile from (e): average height $=58$ $\mathrm{nm}$

Figure 5. Comparison of measurements of the $0.6 \mu \mathrm{m}$ pitch grating through the $24 \mu \mathrm{m}$ diameter microsphere in white light $\left(\mathrm{LS}_{1}, \lambda_{0}=760 \mathrm{~nm}\right)$ with the $50 \mathrm{X}$ Leitz-Linnik microscope and using AFM.

\subsection{Measurements of the $0.4 \mu \mathrm{m}$ pitch grating}

As for the previous grating, the $0.4 \mu \mathrm{m}$ pitch grating can also not be resolved directly, the 3D measurements not being possible just with the objective under the Leitz-Linnik. On the contrary, this grating could be measured through the microsphere, giving a magnification of $x 4.2$ and a measured depth of $55 \mathrm{~nm}$ (figure 6(a), (b), (c)). The AFM measurements reveal a pitch of $0.41 \mu \mathrm{m}$ and a total depth of around $95 \mathrm{~nm}$ (figure 6(d), (e), (f)).

A comparison of the results of the 3D measurements with the different techniques are given in Table 1 for the three grating pitches. These results show an increase in magnification of between 3.65 and 4.5. If we take the best lateral resolution though the microsphere as being half the period of the smallest grating measured, this would be $200 \mathrm{~nm}$ (Table 1), resulting in an increase in resolution by a factor of 4.39 . 


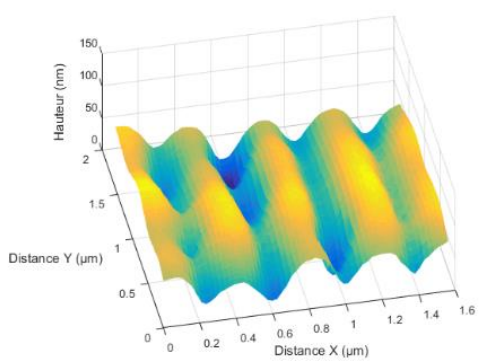

(a) 3D measurement through the microsphere.

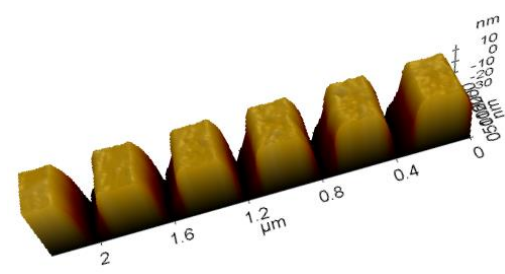

(d) 3D view using AFM.

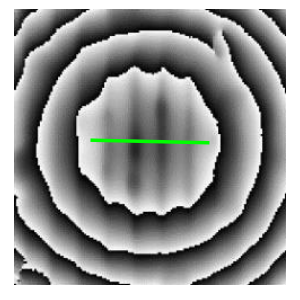

(b) Topography.

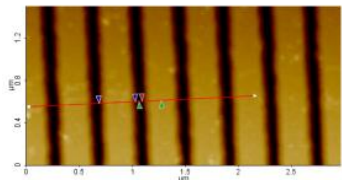

(e) Topography.

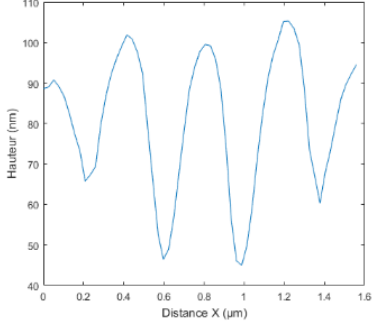

(c) Profile from (b): average height $=55$ nm.

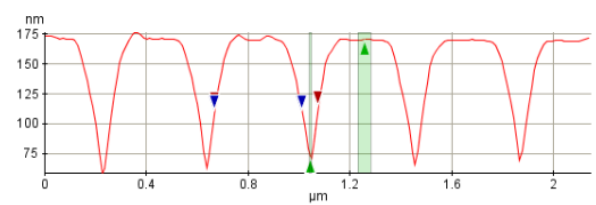

(f) Profile from (e): average height $=95$ $\mathrm{nm}$.

Figure 6. Comparison of measurements of the $0.4 \mu \mathrm{m}$ pitch grating through the $24 \mu \mathrm{m}$ diameter microsphere in white light $\left(\mathrm{LS}_{2}, \lambda_{0}=560 \mathrm{~nm}\right)$ with the $50 \mathrm{X}$ Leitz-Linnik microscope and using AFM.

\subsection{Determination of axial sensitivity}

To determine the noise limited axial sensitivity of the measurements through the microsphere, line profiles over short distances $(1 \mu \mathrm{m})$ were made along the flat ridges of the gratings. The total roughness $\left(R_{t}\right)$ for the $1.2 \mu \mathrm{m}$ pitch grating with the incandescent lamp $\left(\mathrm{LS}_{1}\right)$ was $3.5 \mathrm{~nm}$ (figure $7(\mathrm{a})$ ) and that for the $0.6 \mu \mathrm{m}$ grating with the LED $\left(\mathrm{LS}_{2}\right)$ was $3.3 \mathrm{~nm}$ (figure 7(b)), indicating an axial sensitivity limit of several $\mathrm{nm}$.

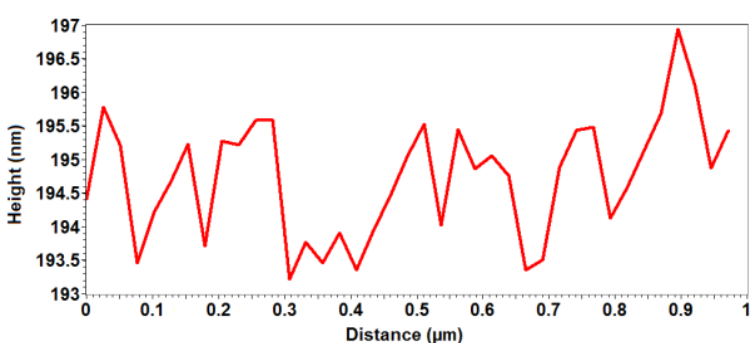

(a) Line profile from a flat ridge on the $1.2 \mu \mathrm{m}$ pitch grating through the microsphere using source $\mathrm{LS}_{1}$ (figure $4(\mathrm{~b})) ; R_{t}=3.5 \mathrm{~nm}$.

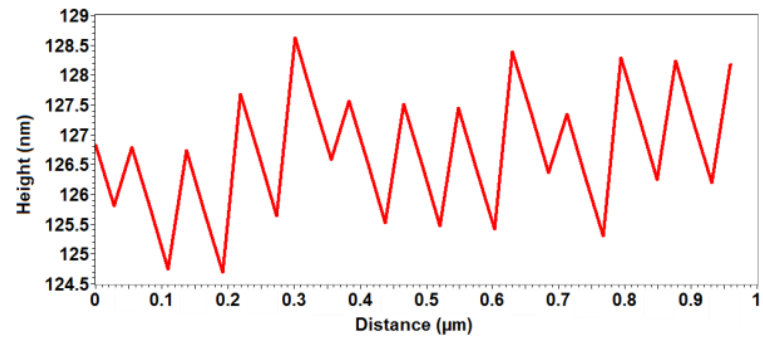

(b) Line profile from a flat ridge on the $0.4 \mu \mathrm{m}$ pitch grating through the microsphere using source $\mathrm{LS}_{2}$ (figure 6(b)); $R_{t}=3.3 \mathrm{~nm}$.

Figure 7. Determination of the noise limited axial sensitivity through the microsphere from the local profiles of flat parts measured with each source $\mathrm{LS}_{1}$ and $\mathrm{LS}_{2}$. 
Table 1. Comparison of results of 3D measurements of RS-N standard gratings using direct interferometry, interferometry with microsphere and AFM. Legend: (xx) cannot be measured.

\begin{tabular}{ccccc|cc}
\hline Grating & Depth & \multicolumn{5}{c}{ Leitz-Linnik WL $\left(\lambda_{0}=\mathbf{7 6 0} \mathbf{~ n m}\right)$} \\
\cline { 3 - 7 } $\begin{array}{c}\text { pitch } \\
(\boldsymbol{\mu m})\end{array}$ & $\begin{array}{c}\text { AFM } \\
(\mathbf{n m})\end{array}$ & \multicolumn{2}{c}{ Direct with objective } & \multicolumn{2}{c}{ Through $24 \boldsymbol{\mu m}$ microsphere } \\
\cline { 3 - 7 } & & $\begin{array}{c}\lambda_{0} \\
(\mathbf{n m})\end{array}$ & $\begin{array}{c}\text { Depth } \\
(\mathbf{n m})\end{array}$ & $\begin{array}{c}\text { Lat res } \\
\boldsymbol{R}_{\boldsymbol{c}}(\mathbf{n m})\end{array}$ & Magnification & Depth $(\mathbf{n m})$ \\
\hline 1.2 & 153 & $760 \mathrm{~nm}$ & 30 & 1190 & 4.5 & 150 \\
\hline 0.6 & 58 & $760 \mathrm{~nm}$ & $\mathrm{xx}$ & 1190 & 3.65 & 46 \\
\hline 0.4 & 95 & $560 \mathrm{~nm}$ & $\mathrm{xx}$ & 877 & 4.2 & 55 \\
\hline
\end{tabular}

\section{RESULTS OF SIMULATIONS}

\section{FEM simulations of imaging properties of a microsphere}

Further understanding of the imaging properties of the microsphere can be gained by carrying out 2D FEM simulations. When a microsphere is illuminated with a plane wave, the photonic nanojet phenomenon occurs below the microsphere as a concentrated intensity with a size that is smaller than the classical diffraction limit ${ }^{13}$. By analogy with a classical lens, the position of the photonic nanojet could be considered as being at the image focus point of the microsphere. This suggests that imaging with a microsphere should also be possible with a lateral resolution limit that is smaller than the classical diffraction spot size ${ }^{8}$. The analogy with geometrical optics also suggests, figure 8(a), the formation of a virtual image. This is in agreement with the experimental observations in which a virtual image is indeed formed. To test this hypothesis, we have studied the interaction between two point sources emitting light at a wavelength in free space of $550 \mathrm{~nm}$ below a 24 $\mu \mathrm{m}$ diameter glass microsphere $(\mathrm{n}=1.5)$ in air (figure $8(\mathrm{~b})$ ) in order to find the point spread function (PSF) of the microsphere. The goal was to determine the resolution limit, the position of the virtual image plane and the magnification. The boundary conditions for the right, left and bottom of the figure are Perfectly Matched Layer (PML) absorbing boundaries.

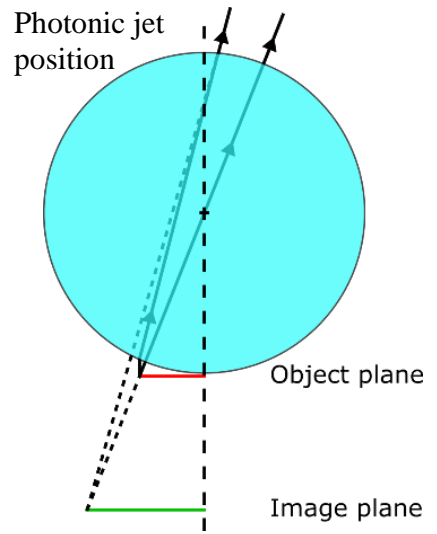

(a) Image magnification through a microsphere.

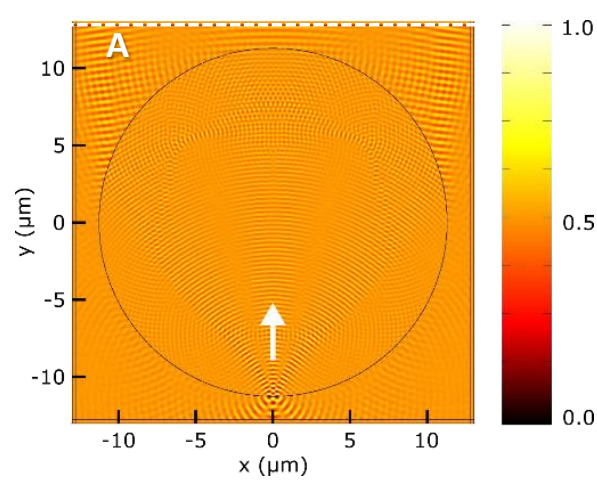

(b) Real part of electric field $|\mathrm{Ez}|$ of the light emitted by two point sources below the microsophere and resulting in the field at the dotted line "A".

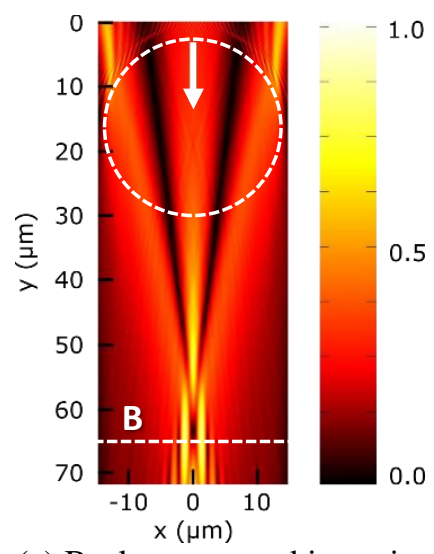

(c) Back propagated intensity without microsphere (previous position indicated by dotted circle) to determine image formation of the point sources (indicated by dotted line at "B").

Figure 8. Determination of the imaging properties through a $24 \mu \mathrm{m}$ glass microsphere using light at a wavelength of $\lambda=550 \mathrm{~nm}$ using 2D FEM simulation in air. White arrows in (b) and (c) indicate direction of propagation. 
The next step is to take the electric field so formed at the line "A" (top of the image in figure 8(b)) and to perform a time reversed propagation of the electric field in free space (i.e. without the microsphere), to determine from which point the outgoing light seems to come, that is the virtual image of the point source that would be observed by the microscope (figure $8(\mathrm{c})$ ). The position of the virtual image plane is then retrieved from the intensity peaks of the image of the two point sources as shown by the line "B". In figure $8(\mathrm{c})$, the position of the image plane is at almost $50 \mu \mathrm{m}$ below the center of the microsphere. From simulation, the magnification is defined as the ratio between the lateral distances of the two intensity peaks and that of the point sources, giving a value of 4.2 in this case. The resolution of the microsphere can then be determined from the Full Width at Half Maximum (FWHM) of the PSF which is defined as the deconvolution of the image with the object, giving a value of $250 \mathrm{~nm}$ in monochromatic illumination. For white light at the same central wavelength, the lateral resolution would be slightly better, as found experimentally (Table 1). A further discussion of the simulations carried out is given in ${ }^{14}$.

\section{CONCLUSIONS}

The goal of the present work was to perform an initial exploration of the 3D super-resolving properties of glass microspheres placed on narrow pitch square profile $\mathrm{Si}$ gratings below the objective of a Linnik interferometer. Experimental measurements and simulations have been carried out on gratings with pitches of $1.2 \mu \mathrm{m}, 0.6 \mu \mathrm{m}$ and $0.4 \mu \mathrm{m}$ measured using phase shifting in white light interferometry. The experimental results demonstrate a lateral super-resolution effect with an increase in the lateral resolution by a factor of 3.97 to 4.39 and the ability to measure the surface topography with an axial sensitivity of several $\mathrm{nm}$. In the experiments carried out in white light, the best resolution obtained in air was $200 \mathrm{~nm}$, allowing the $0.6 \mu \mathrm{m}$ and $0.4 \mu \mathrm{m}$ gratings to be measured that could not be resolved by the objective alone.

The results of the initial simulations help to better understand the imaging properties of the $24 \mu \mathrm{m}$ glass microsphere. They confirm the formation of a virtual image, giving a magnification of about 4.2 and an improvement in lateral resolution of better than 3.5, in agreement with the experimental observations.

While the lateral resolution was observed to be improved through the microsphere, there was nonetheless certain artefacts in the 3D measurements. For example, regions of blurring could be observed, possibly due to non-optimized illumination conditions or non-uniformity or surface damage to the microspheres. While the AFM measurements confirmed most of the structural details of the grooves measured through the microspheres, certain differences remained, possibly due to the test sample being a prototype and displaying slight shape variations in different regions, notably in the grooves.

The results confirm that laterally super-resolved 3D interferometry can be performed in a far field optical imaging system using dielectric microspheres placed on the sample below the imaging objective. This new microsphere-assisted technique opens new possibilities for high resolution characterization in nanometrology.

\section{ACKNOWLEDGEMENTS}

Thanks are extended to Julien Zelgowski for helping in positioning the microspheres.

\section{REFERENCES}

[1] Montgomery P. C., Serio B., Anstotz F. and Montaner D., "Far field optical nanoscopy: how far can you go in nanometric characterization without resolving all the details?," Applied Surface Science 281, pp. 89-95 (2013).

[2] De Groot P., [Handbook of Optical Metrology: Principles and Applications], CRC Press, Boca Raton (2015).

[3] Gustafsson M. G. L, Agard D. A. and Sedat J. W., "I5M: 3D widefield light microscopy with better than $100 \mathrm{~nm}$ axial resolution," J. Microscopy 195, 10 (1999).

[4] Liu H., Bailleul J., Simon B., Debailleul M., Colicchio B., and Haeberlé O., "Tomographic diffractive microscopy and multiview profilometry with flexible aberration correction," Appl. Opt. 53, 748-755 (2014).

[5] Wang Z. B., Guo W., Li L., Luk'yanchuk B., Khan A., Liu Z., Chen Z. and Hong M., "Optical virtual imaging at 50 nm lateral resolution with a white-light nanoscope," Nat. Commun. 2, 218 (2011). 
[6] Wang F., Liu L., Yu P., Liu Z., Yu H., Wang Y. and Li W. J., "Three-Dimensional Super-Resolution Morphology by Near-Field Assisted White-Light Interferometry," Sci Rep. 6, 24703 (2016).

[7] Kassamakov I., Nolvi A. and Hæggström E., "3D Super-resolution Label-free Imaging," Conference on Lasers and Electro-Optics (CLEO) OSA Technical Digest, AM4O.2 (2016).

[8] Montgomery P. C., Lecler S., Leong-Hoï A. and Pfeiffer P., "3D nano surface profilometry by combining the photonic nanojet with interferometry," Journal of Physics Conference Series 794, 012006 (2017).

[9] Montgomery P. C., Montaner D. and Salzenstein F., "Tomographic analysis of medium thickness transparent layers using white light scanning interferometry and XZ fringe image processing," Proc. SPIE 8430, 843014 (2012).

[10] Dubois A., Grieve K., Moneron G., Lecaque R., Vabre L., and Boccara C., "Ultrahigh-Resolution Full-Field Optical Coherence Tomography," Appl. Opt. 43, 2874 (2004).

[11]Debailleul M., Georges V., Simon B., Morin R., and Haeberlé O., "High-resolution three-dimensional tomographic diffractive microscopy of transparent inorganic and biological samples," Opt. Lett. 34, 79-81 (2009).

[12] Li L., Guo W., Yan Y., Lee S. and Wang T., "Label-free super-resolution imaging of adenoviruses by submerged microsphere optical nanoscopy," Light: Sci. \& Appl. 2, e104 (2013).

[13]Lecler S., Takakura Y., and Meyrueis P., "Properties of a three-dimensional photonic jet," Opt. Lett. 30, 2641-2643 (2005).

[14] Perrin S., Leong-Hoi A., Lecler S. and Montgomery P. C., "Role of the coherence in assisted microsphere nanoscopy," Proc. SPIE 10330, to be published (2017). 